\section{Self-Limited Systemic Sarcoidosis in a Pregnant Woman Presenting as Oral Papules}

\section{Keywords}

Oral; Mucosal; Sarcoidosis; Pregnancy

\section{Case Description}

A 30-year-old pregnant woman in her third trimester presented with a few months history of multiple asymptomatic lesions in the oral cavity. She had concomitant recurrent painful lesions over the shins. Her system review was non-revealing. She denied any oral or perioral injections, or exposure to insecticides or other environmental chemicals. Her previous medical history was unremarkable, and her family history was negative for sarcoidosis or other connective tissue diseases.

Oral examination revealed multiple non-tender, wellcircumscribed, semi-firm papules, $0.5-1 \mathrm{~cm}$ in diameter located predominantly over the right lower mucosal lip and to a lesser extent over the upper and left lower mucosal lip. The mucosa overlying the largest papule had a bluish hue (Figure 1). Her physical examination was unremarkable except for multiple erythematous and tender subcutaneous nodules over both shins.

Histological examination from the largest oral lesion showed non-caseating naked epithelioid granulomas filling the submucosa (Figure 2). Special stains including PAS, Giemsa, Acid Fast and Fite stains were non-revealing. Polarizing microscopy was negative. Based on the above findings, the diagnosis of oral sarcoidosis was made. Biopsy from a shin nodule revealed a predominantly septal panniculitis consistent with erythema nodosum.

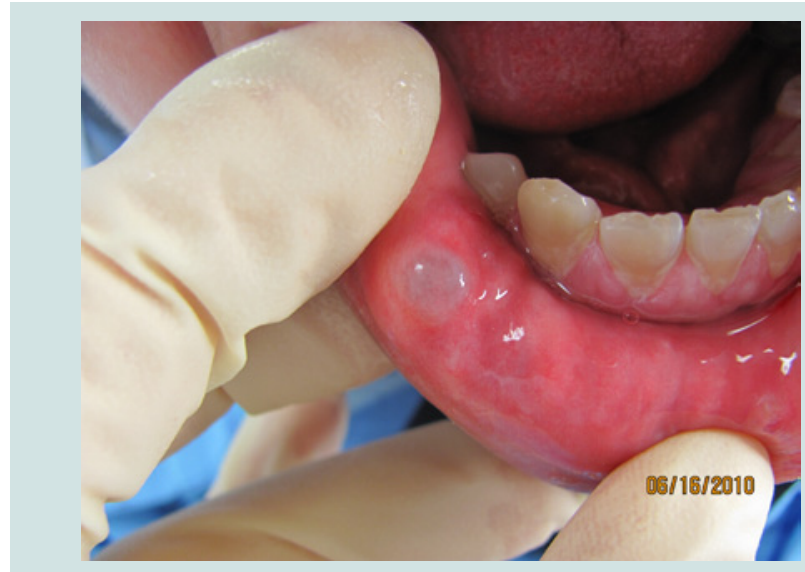

Figure 1: Over the lower lip, a semi-firm submucosal papule with an overlying bluish hue. Multiple adjacent lesions could be palpated.

\section{Journal of}

\section{Clinical \& Investigative} Dermatology

\section{Majed Al Hassanieh ${ }^{1}$, Jinane El Khoury ${ }^{2}$, Ghina Ghaziri $^{3}$ and Samer Ghosn ${ }^{2 *}$}

${ }^{1}$ Department of Internal Medicine, University of Balamand, Beirut, Lebanon

${ }^{2}$ Department of Dermatology, American University of Beirut Medical Center, Beirut, Lebanon

${ }^{3}$ Department of Obstetrics and Gynecology, American University of Beirut Medical Center, Beirut, Lebanon

\section{*Address for Correspondence}

Samer Ghosn, Associate Professor in the Department of Dermatology at the American University of Beirut Medical Center, Riad El Solh St, Beirut, Lebanon, P.O. Box 11-0236, Tel: +961-1-350000, Ext. 5333; Fax: +961-1745320; Email: sg03@aub.edu.lb

Submission: 29 July 2014

Accepted: 10 October 2014

Published: 13 October 2014

Chest X-ray revealed asymmetrical hilar prominence with no evidence of lung nodules (Figure 3). Computed tomography of the chest revealed enlarged mediastinal and hilar lymph nodes, with a few parenchymal and pleural-based lung nodules. Ophthalmological examination was normal

The clinical picture was overall consistent with stage 2 sarcoidosis. The pregnancy was carried to term and the patient delivered without complications. The oral lesions resolved spontaneously within a month after delivery. A follow-up chest X-ray six months later was normal (Figure 4). Yearly clinical and radiographic follow-up did not reveal disease recurrence.

Sarcoidosis is a systemic granulomatous disease affecting mostly young individuals. Oral sarcoidosis, including involvement of the jaw bone and the gingiva, is rare with less than a hundred cases reported [1]. Oral mucosal sarcoidosis not related to salivary glands or lymph nodes is even rarer with around 70 cases reported in the literature, most commonly involving the buccal mucosa, followed by the gingiva, lip, tongue and palate [2-4]. There is a slight female predilection with a reported age distribution ranging from 5 to 72 years [1-3]. Like in our case, most patients with mucosal involvement present with nontender circumscribed submucosal papulonodules but the disease may present as superficial ulcerations [2-5]. Although oral mucosal sarcoidosis is usually considered a manifestation of chronic systemic sarcoidosis $[2,6]$, two studies showed that only $35 \%$ and $58 \%$ of oral mucosal sarcoidosis cases were associated with systemic disease $[1,2]$. The current case illustrates that systemic sarcoidosis may rarely present with oral lesions. In fact, from all orally involved sarcoidosis cases, only one-third of the patients presented with oral lesion as the first manifestation of the disease [7]

Clinically, the lesions may mimic bite fibroma, mucocele, angiolymphoid hyperplasia, nodular amyloidosis and other infiltrative disorders. The histological differential diagnosis includes orofacial granulomatosis, Crohn's disease, foreign body reaction, and chronic atypical bacterial and fungal infections. Clinicopathological correlation and proper histochemical staining should be obtained to reach a correct diagnosis. 


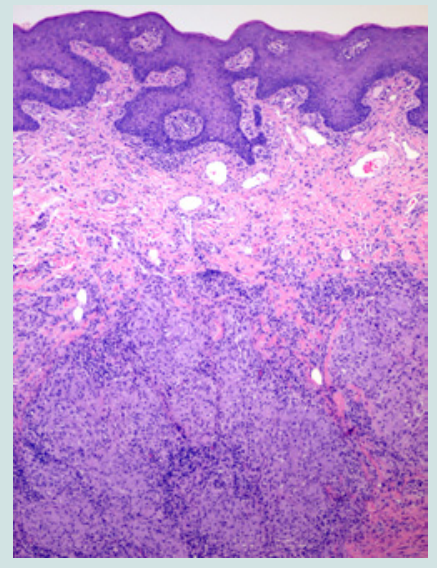

Figure 2: Sarcoidal granulomatous dermatitis.

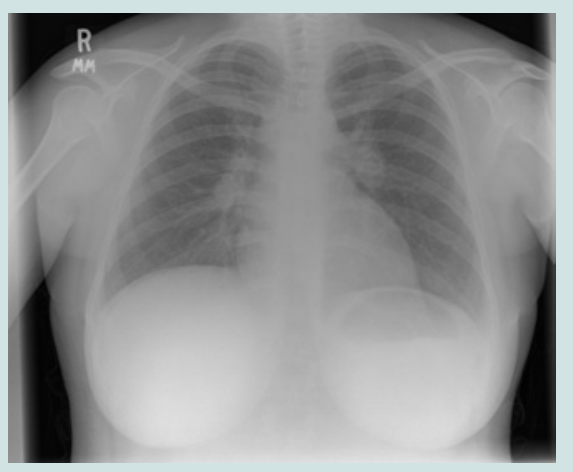

Figure 3: Chest X-ray AP view during pregnancy showing the asymmetrical hilar prominence with no evidence of lung nodules.

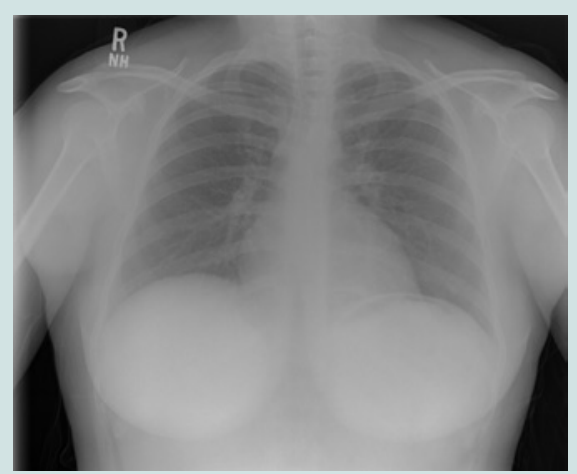

Figure 4: Chest X-ray AP view 6 months later showing clear lungs in comparison with the previous image.

In pregnant women, both flaring and remission of sarcoidosis have been reported. Most studies, however, suggest that sarcoidosis generally portends a good prognosis during pregnancy. This has been partly attributed to the gestational synthesis of steroids by the adrenal glands [8,9]. Mayock et al. observed the course of 16 pregnancies in 10 patients with sarcoidosis over eight years. In their study, pregnancy had a beneficial effect on sarcoidosis in 8 patients that was lost postpartum in 4 patients in a rebound-relapse phenomenon. In this same study, sarcoidosis has been associated with miscarriage in 2 cases, and some rare fetal abnormalities like polydactyly [9]. Another study by Chapelon et al. analyzed the evolution of sarcoidosis during 33 pregnancies in 11 women and showed no relapse of inactive sarcoidosis during pregnancy and variable course in patients with active sarcoidosis at the beginning of pregnancy. In this same study, 23 out of 33 pregnancies led to the birth of healthy babies. The remaining 10 ended up with abortion (spontaneous, voluntary, therapeutic) with the major event being fetal hypotrophy in six cases [10]. Hadid et al. conducted a population-based retrospective cohort study to compare women with and without sarcoidosis at delivery. Their study showed that women with sarcoidosis were more likely to have preeclampsia, eclampsia, deep vein thrombosis, pulmonary embolism, and premature delivery. There was also an increased risk of caesarean deliveries and postpartum hemorrhages [11].

The current case is unusual in that the patient was not known to have sarcoidosis before her pregnancy. Sarcoidosis is most frequent in women of reproductive age, and therefore the appearance of sarcoidosis in pregnant women should not be rare. However, the diagnosis of sarcoidosis during pregnancy is difficult since radiography is often avoided and may be delayed up to 12 months postpartum. Like the previous reports, the current case confirms that sarcoidosis developing in pregnancy is usually associated with good prognosis [8]. The disease in this case regressed spontaneously shortly after delivery and never recurred within 3 years of follow-up. The "self limited" nature of this patient's sarcoidosis could also be attributed to the associated erythema nodosum, a well-known good prognostic feature in the setting of systemic sarcoidosis.

Current guidelines for further follow up on sarcoidosis patients with inactive disease is a yearly monitoring for at least 3 years, whereas those with active disease need a regular follow up at 3-6 month interval depending on the severity of the disease. Surveillance ranges from a thorough history and physical exam to chest imagining and PFT. Asymptomatic patients with persistent radiographic changes are followed indefinitely for recurrence of the disease. Finally, yearly ophthalmic examination is recommended regardless of eye involvement [12].

Therapeutic indications are the same as outside pregnancy, systemic/ intralesional corticosteroid therapy being the only proven treatment during this period. Methotrexate and antimalarial drugs are contraindicated. In many instances however, the lesions may regress spontaneously [13].

In conclusion, sarcoidosis should be considered in the differential of oral mucosal lesions. To the best of our knowledge, we describe the first case of oral mucosal sarcoidosis as the first manifestation of systemic sarcoidosis in a pregnant woman. The often-benign course of this systemic disease during pregnancy should be taken into consideration when tailoring a therapeutic plan. Sarcoidosis may be difficult to diagnose during pregnancy and may pose deleterious effect on the patient, as well as the fetus.

\section{References}

1. Suresh L, Radfar L (2005) Oral sarcoidosis: a review of literature. Oral dis 11 : 138-145.

2. Kasamatsu A, Kanazawa H, Watanabe T, Matsuzaki O (2007) Oral sarcoidosis: report of a case and review of literature. J Oral Maxillofac Surg 65: 1256-1259. 
Citation: Al Hassanieh M, El Khoury J, Ghaziri G, Ghosn S. Self-Limited Systemic Sarcoidosis in a Pregnant Woman Presenting as Oral Papules. J Clin Investigat Dermatol. 2014;2(3): 3.

3. Bouaziz A1, Le Scanff J, Chapelon-Abric C, Varron L, Khenifer S, et al (2012) Oral involvement in sarcoidosis: report of 12 cases. QJM: 105:755-67.

4. Motswaledi M, Khammissa R, Jadwat $\mathrm{Y}$, Lemmer J, Feller L (2014) Ora sarcoidosis: a case report and review of the literature. Aust Dent J 59:389394.

5. Kolokotronis AE, Belazi MA, Haidemenos G, Zaraboukas TK, Antoniades DZ (2009) Sarcoidosis: oral and perioral manifestations. Hippokratia 13: 119 121

6. Serrat Soto A, Lobo Valentin P, Redondo Gonzalez LM, Sanz Santa Cruz C Verrier Hernandez A (1997) Oral sarcoidosis with tongue involvement. Oral Surg Oral Med Oral Pathol Oral Radiol Endod 83: 668-671.

7. Al-Azri AR, Logan RM, Goss AN (2012) Oral Lesion as the first Clinical Presentation in Sarcoidosis: A Case Report. Oman Med J 27: 243-245.

8. Miloskovic $\vee(2005)$ [Sarcoidosis in pregnancy--diagnostic, prognostic and therapeutic problems]. Med Pregl 58 Suppl 1: 51-54.
9. Mayock RL, Sullivan RD, Greening RR, Jones R Jr (1957) Sarcoidosis and pregnancy. J Am Med Assoc 164: 158-163.

10. Chapelon Abric C, Ginsburg C, Biousse V, Wechsler B, de Gennes C, et al. (1998) [Sarcoidosis and pregnancy. A retrospective study of 11 cases]. Rev Med Interne 19: 305-312.

11. Hadid V, Patenaude V, Oddy L, Abenhaim H (2014) Sarcoidosis and pregnancy: obstetric and neonatal outcomes in a population-based cohort. Obstet Gynecol 123 Suppl 1: 148S.

12. Amin EN, Closser DR, Crouser ED (2014) Current best practice in the management of pulmonary and systemic sarcoidosis. Ther Adv Respir Dis 8: 111-132.

13. Ellafi M, Valeyre D (1999) [Sarcoidosis and pregnancy]. Rev Pneumol Clin 55:335-337.

\section{Copyright}

(c) 2014 Hassanieh MA, et al. This is an open access article distributed under the Creative Commons Attribution License, which permits unrestricted use, distribution, and reproduction in any medium, provided the original work is properly cited. 